\title{
A hexanucleotide repeat expansion in C9ORF72 links amyotrophic lateral sclerosis and frontotemporal dementia
}

\begin{abstract}
An expanded GGGGCC hexanucleotide repeat in the C9ORF72 gene on chromosome 9p21 provides a genetic link between amyotrophic lateral sclerosis (ALS) and frontotemporal dementia (FTD), according to two independent reports in Neuron.
\end{abstract}

Though traditionally considered to be separate conditions, ALS and FTD are increasingly being recognized as belonging to a disease continuum, with common features such as the presence of TDP-43positive inclusions in the CNS. Moreover, a substantial number of individuals exhibit a combined ALS-FTD phenotype.

A risk locus for ALS and FTD on the short arm of chromosome 9 was first identified through linkage analysis studies reported in 2006, and subsequent work has brought us ever closer to locating the causative mutation. "In 2010, we published a genome-wide association study of ALS in Finland, which narrowed the region of interest from a 7.7 $\mathrm{Mb}$ region down to only $232 \mathrm{~Kb}$," explains Bryan Traynor from the National Institute on Aging, USA, who is the corresponding author of one of the new studies. "This is the equivalent in genomics terms of going from a city down to a street."

Traynor and colleagues had previously identified an ALS-FTD phenotype linked to chromosome 9p21 in two families, one from the UK and one from the Netherlands. To pinpoint the exact genetic defect, his team performed next-generation sequencing on samples of chromosome 9 DNA from these families, as well as from a Finnish cohort with 9p21-associated ALS. This approach uncovered an expanded GGGGCC repeat in a noncoding region (intron 1) of C9ORF72, a gene of as yet unknown function that is highly conserved between species.

In a separate study, a group led by Rosa Rademakers from the Mayo Clinic Florida, USA sequenced DNA from individuals belonging to the VSM-20 (Vancouver, San Francisco, and Mayo family 20) kindred-a large family with autosomal dominant ALS-FTD. By sequencing the coding and noncoding regions of five genes in the chromosome 9p candidate region, Rademakers' team independently identified the same hexanucleotide repeat that was found by Traynor and colleagues. Her team followed up on a finding of apparently nonMendelian inheritance, which led them to discover the repeat expansion.

"We also performed RNA fluorescent in situ hybridization using a probe specifically designed to bind RNA fragments containing GGGGCC repeats," says Rademakers. "Using this technique, we were able to show nuclear RNA foci in the frontal cortex and spinal cord tissue of of patients with expanded repeats in the C9ORF72 gene."

As the authors of both studies point out, the C9ORF72 repeat expansion is the most frequent cause of inherited ALS and FTD to be identified so far. In addition, the repeat expansion has been observed in a significant number of cases of sporadic FTD and ALS.

The new findings have particular relevance to the Finnish population, which shows the highest incidence of ALS worldwide. "We found that the hexanucleotide repeat expansion in C9ORF72 accounts for around $50 \%$ of ALS in Finland," says Traynor. "What we were not expecting was that the same repeat expansion was going to account for such a high percentage of familial ALS cases - 38\% - outside of Finland in outbred European-descent individuals."

The research also offers insights into the molecular mechanisms underlying the development of ALS and FTD, lending particular support to a role for defects in RNA processing. "We provide evidence for the generation of toxic RNA foci as a result of GGGGCC repeat expansions," explains Rademakers. "This GC-rich mRNA could cause disease by recruiting and sequestering RNA-binding proteins into the foci, leading to aberrant splicing of RNAs."

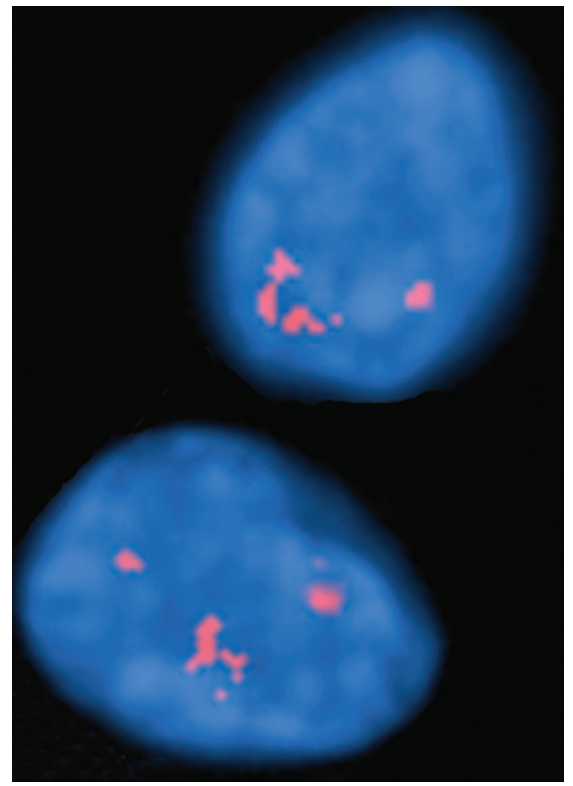

RNA foci, visualized using a Cy3-labeled (GGCCCC) oligonucleotide probe (red), in the nuclei of two lower motor neurons from an ALS-FTD patient carrying the expanded GGGGCC repeat. Courtesy of Dr Rosa Rademakers.

The data open up a number of avenues for future investigation. For example, why do some patients with the C9ORF72 repeat expansion develop ALS while others develop FTD or a combination of the two conditions? Also, is this mutation involved in any other neurodegenerative diseases?

"This work greatly facilitates future development of cell-based assays and animal models that can be used to screen therapeutic agents," says Traynor. He warns, however, that "we have to be careful not to falsely raise the expectations of patients - in the past, it has typically taken 10 years to go from drug discovery to clinical trials."

Heather Wood 